\title{
Lrig1 and Wnt dependent niches dictate segregation of resident immune cells and melanocytes in murine tail epidermis
}

\author{
Susanne C. Baess ${ }^{1,2,3}$, Annika Graband ${ }^{1,2,3}$, Kristin Seré ${ }^{4,5}$, Martin Zenke ${ }^{4,5}$, \\ Catherin Niemann ${ }^{3,6}$ and Sandra Iden ${ }^{1,2,3 \#}$
}

${ }^{1}$ Cell and Developmental Biology, Center of Human and Molecular Biology (ZHMB), Saarland University, Faculty of Medicine, Homburg/Saar, Germany

${ }^{2}$ Cologne Excellence Cluster on Cellular Stress Responses in Aging-Associated Diseases (CECAD), University of Cologne, Germany

${ }^{3}$ Center for Molecular Medicine Cologne (CMMC), University of Cologne, Germany

${ }^{4}$ Institute for Biomedical Engineering, Department of Cell Biology, RWTH Aachen University Medical School, Aachen, Germany

${ }^{5}$ Helmholtz-Institute for Biomedical Engineering, RWTH Aachen University, Aachen, Germany ${ }^{6}$ Center of Biochemistry, Faculty of Medicine and University Hospital Cologne, Germany

\#Correspondence: sandra.iden@uks.eu

Lead contact: Sandra Iden, Cell and Developmental Biology, Saarland University, Faculty of Medicine, Kirrberger Strasse, Building 61.4, 66421 Homburg/Saar, Germany, sandra.iden@uks.eu, ORCID: 0000-0003-2333-9827

Running title: Patterning skin functions

Keywords: epidermis, skin, tissue patterning, Langerhans cell, immune cell, melanocyte, Wnt signaling, intercellular communication. 


\section{ABSTRACT}

The barrier-forming, self-renewing mammalian epidermis comprises keratinocytes, pigmentproducing melanocytes, and resident immune cells as first-line host defense. In murine tail skin, interfollicular epidermis patterns into pigmented 'scale' and non-pigmented 'interscale' epidermis. Why and how mature melanocytes confine to scale epidermis is unresolved. Here, we delineate a cellular hierarchy among epidermal cell types that determines skin patterning. Already during postnatal development, melanocytes co-segregate with newly forming scale compartments. Intriguingly, this process coincides with partitioning of both Langerhans cells and dendritic epidermal T-cells to interscale epidermis, suggesting functional segregation of pigmentation and immune surveillance. Analysis of non-pigmented mice and of mice lacking melanocytes or resident immune cells revealed that immunocyte patterning is melanocyte- and melanin-independent, and, vice versa, immune cells do not control melanocyte localization. Instead, genetically enforced progressive scale fusion upon Lrig1 deletion showed that melanocytes and immune cells dynamically follow epithelial scale:interscale patterns. Importantly, disrupting Wnt-Lef1 function in keratinocytes caused melanocyte mislocalization to interscale epidermis, implicating canonical Wnt signaling in organizing the pigmentation pattern. Together, this work uncovered cellular and molecular principles underlying the compartmentalization of tissue functions in skin.

\section{SUMMARY STATEMENT}

Pigmentation and immune surveillance functions in murine tail skin are spatially segregated by Lrig1- and Wnt-Lef1-dependent keratinocyte lineages that control the partitioning of melanocytes and tissue-resident immune cells into distinct epidermal niches. 


\section{INTRODUCTION}

The skin acts as dynamic interface between the organism and its environment. It protects from uncontrolled water loss and external threats such as pathogens, toxins, mechanical damage and temperature variation. Its outermost stratified epidermis is continuous with epidermal appendages like hair follicles and sweat glands (Chuong and Noveen, 1999). Proliferative, undifferentiated basal layer keratinocytes (KCs) attach to the underlying basement membrane, and, following asymmetric cell division or delamination, progressively differentiate to constitute the spinous, granular and cornified layers (Blanpain and Fuchs, 2006; Dias Gomes and Iden, 2021; Gonzales and Fuchs, 2017; Ray and Lechler, 2011). The epidermis also hosts other resident cell types with crucial functions for the organism: neural-crest derived melanocytes (MCs) provide melanin for hair colorization and to protect KCs from UV damage (D'Orazio et al., 2013), whereas Langerhans cells (LCs) and dendritic epidermal T-cells (DETCs) constitute a first line of defence against environmental pathogens and malignant transformation in murine skin (Deckers et al., 2018; MacLeod et al., 2013). Various UV-induced KC-derived soluble factors have been implicated in induction of melanin synthesis in MCs (Upadhyay et al., 2021). Yet, how KC, LC, DETC and MC networks are spatiotemporally coordinated within the densely packed epidermis to simultaneously ensure skin barrier, pigmentation and host defence is largely unresolved.

The interfollicular epidermis (IFE) of murine ear and back-skin harbours evenly distributed immune cells (Bergstresser et al., 1980) and is largely devoid of MCs, which mostly reside in the dermis (Hirobe, 1984; Kunisada et al., 2000). Tail skin instead exhibits two distinct epidermal compartments: 'Scale' IFE forms a spherical epidermal patch just above hair-follicle triplets and undergoes parakeratotic differentiation, characterized by lack of a granular layer and nucleated cornified layer KCs. 'Interscale' IFE surrounds the scale IFE and, alike ear and back-skin IFE, displays orthokeratotic differentiation with a granular layer and enucleated cornified layer KCs (Didierjean et al., 1983). Gomez et al. (2013) demonstrated that epidermal lineages of scale and interscale IFE express unique marker genes and are regulated by Wnt, Edaradd, and Lrig1. Strikingly, these intraepidermal patterns in tail skin correlate with a strict compartmentalization of LCs, which populate the interscale IFE (Schweizer and Marks, 1977), and of pigmented MCs, which localize to the scale IFE where they persist throughout adulthood (Glover et al., 2015; Gomez et al., 2013). Further, a small population of amelanotic, quiescent MCs in interscale IFE has been reported (Glover et al., 2015; Köhler et al., 2017). How such segregation of epidermis-resident cells is achieved and maintained is poorly understood. Gomez et al. (2013) showed that MCs are dispensable for scale IFE formation; however, molecular and cellular signals orchestrating the mutually exclusive MC:immunocyte distribution remain to be identified. It is currently not known whether scale-based MCs antagonize the localization of immunocytes (or vice versa). Mouse scale IFE closely resembles the epidermal 
MC distribution of human skin and hence is used to study mechanisms of skin pigmentation (Fitch et al., 2003; Van Raamsdonk et al., 2004) and intraepidermal melanoma (Köhler et al., 2017). Gaining insight into heterologous communication between different epidermis-resident cell types will thus be beneficial to better understand human skin physiology and disease. In this study, we therefore set out to reveal potential interactions and interdependencies of KCs, MCs and immune cells regarding functional compartmentalization in murine tail skin.

\section{RESULTS AND DISCUSSION}

\section{DETCs, LCs and MCs reside in distinct compartments of the tail IFE}

To first map the localization of DETCs, LCs and MCs with respect to IFE niches we performed immunohistochemistry on tail epidermis whole mounts of 6-15 week old C57BL/6 mice. Coimmunostaining for Keratin-31 (K31) marked parakeratotic differentiation and hence scale IFE in epidermal tail whole-mounts (Gomez et al., 2013). In agreement with earlier reports, Trp2/DCT-positive MCs localized to the scale IFE (Glover et al., 2015; Gomez et al., 2013) whereas CD207/Langerin-positive LCs were confined to the adjacent K31-negative interscale IFE (Schweizer and Marks, 1977)(Fig. 1A,D). Intriguingly, co-immunostaining of $\gamma \delta$ TCR (marking DETCs) and Langerin revealed that DETCs and LCs co-distribute within the interscale IFE, both being excluded from scale IFE (Fig. 1B). Together, these data underpin a remarkable separation of epidermal pigmentary units and resident immune cells in tail IFE, opening the possibility that the scale IFE is an immune-privileged niche. To shed light into establishment of these MC:immunocyte patterns we next examined early postnatal tissues at the onset of scale formation (Gomez et al., 2013). At postnatal day (P) 5, MCs were already prominently enriched at sites of scale induction (Fig. 1C), a phenomenon that reinforced towards P10 (Fig.1C). This indicated that MCs co-segregate with the parakeratotic lineage early during skin development. MHCII immunostaining served to visualize LCs at P5 as Langerin is weakly expressed at these stages (Tripp et al., 2004). MHCII, similar to Langerin, is solely found on LCs in unchallenged epidermis, enabling their identification (Tsuruta et al., 1999). Albeit low in numbers due to the postnatal LC proliferation burst yet to occur (ChangRodriguez et al., 2005; Kobayashi et al., 1987), LCs were confined to interscale IFE in P5 tail epidermis (Fig. 1C). This mutually exclusive distribution of MCs and LCs became even more pronounced with further scale expansion (P10 to P21; Fig. 1C), culminating in the dense LC networks in interscale and MC networks in scale IFE observed in adult tail skin (Fig. 1A,D). These data thus demonstrate that the formation of IFE compartments coincides with the patterned distribution of MCs and epidermis-resident immune cells.

\section{Distinct IFE localization of MCs and immune cells is not interdependent}

We next aimed to dissect a potential hierarchy underlying the patterning of MCs, LCs, DETCs and $\mathrm{KC}$ lineages. The role of resident immune cells in patterning of the other cell types was 
analyzed using mice deficient for the transcription factor Id2, which are characterized by complete LC and DETC loss by young adulthood (Hacker et al., 2003; Seré et al., 2012; Yokota et al., 1999). As expected, LCs were undetectable in adult Id2KO tail epidermis (Fig. 2A). The scale:interscale IFE pattern of mutant mice, however, was comparable to control mice (Fig. $\mathrm{S} 1 \mathrm{~A}$ ), and MCs were confined to scale IFE at the age of 3 months (Fig. 2A,B) up to one year (S.C. Baess, unpublished observation). This suggests that both MC distribution and KC lineage progression into scale and interscale identity are independent of resident immune cells. We cannot formally rule out that a transient presence of immune cells during ontogeny might contribute to initial MC and IFE patterning. Nevertheless, our data clearly demonstrate that LCs and DETCs are not required for scale confinement of MCs throughout adulthood.

Next we asked if, vice versa, MCs antagonize the localization of IFE-resident immune cells. Insects use melanin as innate immune defense (Gillespie et al., 1997), and immunomodulatory roles have also been proposed for mammalian MCs and MC-derived melanin (Burkhart and Burkhart, 2005; Hong et al., 2015). LCs can take up melanin (Breathnach and Wyllie, 1965; Mishima, 1966; Tobin, 1998), which may cause LC emigration to draining lymph nodes (Hemmi et al., 2001). We therefore explored if MCs, or melanin levels, counteract immune cell localization to scale IFE. Comparison of adult pigmented C57BL/6 mice and non-pigmented (albino) FVB/N mice, however, revealed no difference of $\mathrm{MC}$ and $\mathrm{LC}$ distribution per scale:interscale unit (Fig. 2C-E). Similarly, localized spontaneous depletion of MCs and subsequent loss of pigmentation in tail epidermis of C57BL/6 mice (Fig. 2F) did not cause redistribution of LCs or DETCs into the MC-free scale IFE (Fig. 2G,H; Fig. S2A). Together, epidermal MCs and resident immune cells do not affect each other's distribution in tail epidermis.

\section{MC:immunocyte distribution follows scale:interscale patterning}

Our observations and the strikingly similar dynamics of scale formation and MC clustering during postnatal development (Fig. 1C) prompted us to investigate the role of parakeratotic scale and orthokeratotic interscale IFE compartments for immunocyte and MC patterning. Scale progenitors divide more rapidly than interscale progenitors (Gomez et al., 2013; Sada et al., 2016), with spatial control of scale progenitor proliferation likely contributing to scale:interscale patterning and boundary formation. Lrig1, a negative regulator of EGFR signaling, is expressed in the dermis underneath interscale IFE where it is thought to antagonize scale fate and size through dermal-epidermal signaling (Gomez et al., 2013). Upon constitutive Lrig1deletion, initial scale induction is normal but scale IFE compartments progressively enlarge and eventually laterally fuse into wide dorsoventral bands in adult mice (Gomez et al., 2013)(Fig. 3A). Interestingly, analysis of Lrig1KO mice from P10 to adult stages unraveled that, concomitant with lateral scale fusion, MCs assumed a band-like expansion 
corresponding to the K31-labelled scale band, whereas LCs and DETCs were restricted to the diminished interscale stripe (Fig. 3A-C, Fig. S4A). These data indicate that patterning of MCs and immune cells is primarily determined by compartmentalization of the epidermis into scale and interscale IFE.

\section{Defective epidermal Wnt-Lef1 signaling causes MC redistribution to interscale IFE}

We next set out to identify intraepidermal factors mediating $M C$ and immune cell patterning. Among candidate pathways, we hypothesized that canonical Wnt signaling could be involved as it is known for steering epidermal lineage selection in postnatal epidermis (Watt and Collins, 2008) and because Lef1, a key transcriptional effector of canonical Wnt signaling, is predominantly expressed in scale IFE (Gomez et al., 2013; Niemann et al., 2002). Expression of N-terminally truncated Lef1 in epidermal KCs (K14 NNLef1; Niemann et al., 2002), acting as dominant negative inhibitor of canonical Wnt signaling, results in impaired scale differentiation and size with irregular scale:interscale patterning (Gomez et al., 2013). Strikingly, analysis of skin-resident cell types in these mice revealed that MC distribution was largely inverted compared to control mice, with MCs localizing to interscale IFE and to the scale periphery, whereas the scale center was devoid of MCs (Fig. 4A-C). Congruent with this, whereas MCs were rarely found in control ear epidermis, MC numbers were significantly increased in K14 $\Delta$ NLef1 ear epidermis (Fig. 4D,E). These combined findings in tail and ear epidermis suggest that inhibition of canonical Wnt signaling renders orthokeratotic epidermis permissive for MC colonization. To understand if such MC mislocalization is a progressive event, we next assessed the distribution of resident cell types in mutant tissues during postnatal development. K31 immunolabelling of P5 and P10 tail epidermis illustrated that scale formation in K14 $\Delta$ NLef1 mice was delayed when compared to control mice (Fig. 4F)(Gomez et al., 2013). Importantly, already at this early stage MCs failed to cluster to scale IFE and instead overlapped with LCs and DETCs in the K31-negative compartment in K14 $\Delta$ NLef1 mice early-on (Fig. 4F,G). This indicates that canonical Wnt signaling in KCs orchestrates not only scale formation and maintenance but also the distinct patterning of epidermis-resident MCs and hence tail skin pigmentation. Notably, resident immune cells remained confined to the interscale IFE in K14 1 NLef1 mice (Fig. 4A,H), whereby densities of DETCs, but not LCs, were reduced in the ear epidermis of mutant mice (Fig. S3A-D).

Collectively, these findings demonstrate that the mutually exclusive localization of MCs and epidermis-resident immune cells critically depends on epidermal patterns that are orchestrated by antagonistic signaling downstream of Lrig1 and Wnt-Lef1 (Fig. S4A). Our work identified epidermal KCs at the top of a cellular hierarchy that guides the formation and maintenance of functionally distinct tail IFE compartments. 


\section{Conclusion}

Evolutionarily, pigmentation of scale IFE may have prevailed to protect the underlying hair follicle stem cells from UV-mediated damage. Yet, it is intriguing that immune cells are strictly excluded from this compartment. Melanin synthesis involves generation of cytotoxic intermediates, increasing oxidative stress (Denat et al., 2014; Graham et al., 1978; Urabe et al., 1994); however, we show that the epidermal lineages, but not MCs or melanin, determine LC/DETC patterning. KC-derived paracrine factors (e.g., $\alpha$-melanocyte stimulating hormone, endothelin, TGF $\beta$ ) regulate follicular MC stem cell maintenance (Nishimura, 2011) and induction of melanin synthesis ( $\mathrm{Li}$ et al., 2020; Upadhyay et al., 2021). In contrast, principles of KC:immunocyte communication are much less understood. In adult ear epidermis, LCs and DETCs were recently shown to actively maintain a non-random distribution that depends on KC density and engages LC-expressed Rac1 (Park et al., 2021). However, the molecular mechanism how KCs control resident immune cells in this tissue remain unknown. Others reported mRNA transfer from KCs to MCs through exosomes (Cicero et al., 2015; Liu et al., 2019) and from LCs to KCs and DETCs, likely through tunneling nanotubes (Su and Igyártó, 2019), opening the possibility that heterologous cell types in the epidermis actively communicate via such structures. Yet, these studies utilized in vitro systems or non-patterned orthokeratotic epidermis (e.g., ear skin), which leaves open the question of which molecules and signaling pathways mediate the partitioning of epidermis-resident cells to different epidermal niches. Our work identifies an antagonistic framework in which Lrig1-dependent IFE lineage commitment instructs the segregation of epidermal MCs, LC and DETCs, and where epidermal Wnt-Lef1 signaling restricts mature MCs to scale IFE. Further work is necessary to reveal Lef1 transcriptional targets that control MC scale localization. Albeit hypothetical at this

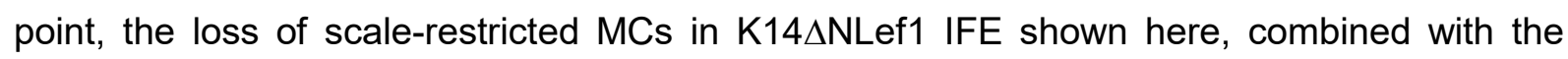
reported high expression of endogenous Lef1 in wild-type scale IFE (Gomez et al., 2013; Niemann et al., 2002), suggests that canonical Wnt signaling elicits expression of MC-retention factors in the scale IFE, rather than inducing MC-repelling factors in adjacent interscale IFE.

We were also intrigued by previous reports of differential transformation susceptibility of scale and interscale IFE cells: Oncogenic hedgehog signalling causes basal cell carcinoma predominantly in interscale IFE (Sánchez-Danés et al., 2016), whereas oncogenic Brafinduced melanomas arise from pigmented MCs in scale IFE (Köhler et al., 2017). This poses the question whether such differential oncogenic outcome is, next to cell-intrinsic factors like proliferative capacity, also linked to the functional separation of pigmentation and skin immunity. LCs sample environmental antigens (Cho et al., 2010; de Jong et al., 2010; Leclercq and Plum, 2021), present self-antigens to naïve T cells to induce tolerance (Stoitzner et al., 2006), and detect altered self-antigens or neo-antigens following external stresses or during 
malignant transformation (Cao et al., 2007; Schreurs et al., 2000). The role of LCs in various skin cancers and wound healing, however, remains controversial, likely due to recently identified additional dermal Langerin-positive non-LC populations that require reinterpretation of data from genetic models with langerin promoters (Deckers et al., 2018; Sheng et al., 2021; Li et al., 2021). DETCs instead have been clearly implicated in immune surveillance, shown to protect against skin tumors and to promote wound healing (Girardi et al., 2003; Havran and Jameson, 2010; Jameson et al., 2002; Kaminski et al., 1993; Schuhmachers et al., 1995; Xiang et al., 2020). Albeit unclear whether above immunocyte functions also apply to tail skin, this opens the possibility that immune surveillance in interscale IFE, and lack thereof in scale IFE, is decisive for tumorigenesis. Interestingly, MCs themselves may serve as non-professional antigen presenting cells (Le Poole et al., 1993a), perform phagocytosis (Le Poole et al., 1993b) and produce immune-regulatory cytokines and chemokines (Hong et al., 2015). Though requiring further investigation, considering that scale IFE lacks resident immune cells, it seems possible that $\mathrm{MCs}$, in principle, compensate for certain immune cell functions in this compartment. Together, dissecting the cellular complexity of epidermal niches and communication of the different resident cell types is an important future task to advance our understanding of cell transformation and malignant progression in the skin.

\section{MATERIALS AND METHODS}

\section{Mice}

All animal breeding and tissue analyses were performed according to institutional guidelines and in compliance with the German federal law for animal protection under control of the North Rhine-Westphalian State Agency for Nature, Environment and Consumer Protection (LANUV, NRW, Germany; file references 81-02.04.2018.A384 and 81-02.04.2018.A401) and the Veterinary Office City of Cologne, Germany (file references UniKöln_Anzeige §4.17.018 and

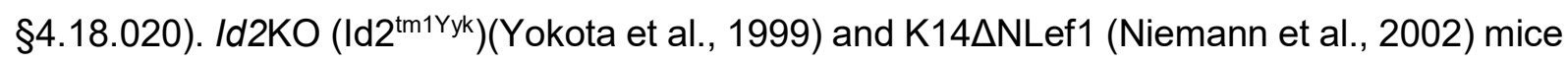
have been previously described. Lrig1KO mice were generated by crossing Lrig1CreERT2/EGFP mice (Page et al., 2013) (kindly provided by Prof. Kim B. Jensen, University of Copenhagen, Danstem, Denmark) on 129 background to homozygosity, resulting in Lrig1 deletion.

\section{Immunohistochemistry of tail epidermal whole-mounts}

Preparation and staining of mouse tail whole-mounts was performed as previously described (Braun, 2003). Briefly, mouse tail skin was peeled off the bone and incubated in $5 \mathrm{mM}$ EDTA/PBS for $2 \mathrm{~h}$ at $\mathrm{RT}$ with mild shaking at $400 \mathrm{rpm}$. After replacing half of the EDTA/PBS volume, the tissue was incubated for another $2 \mathrm{~h}$. Next, the epidermis was separated from the dermis leaving hair follicles attached to the epidermal sheet. Depilation was performed to 
eliminate autofluorescence caused by hair. The epidermis was fixed in pre-cooled acetone for 30 min on ice. For blocking, samples were incubated in PB buffer $(0.5 \%$ skim milk powder, $0.25 \%$ fish skin gelatin (Sigma-Aldrich), 0.5\% Triton-X100 in HBS (20 mM HEPES pH 7.2, $0.9 \% \mathrm{NaCl})$ ) for $1 \mathrm{~h}$ at RT followed by in incubation with primary antibodies diluted in PB buffer over-night at RT. The next day samples were extensively washed for $6-8 \mathrm{~h}$ with $0.5 \%$ Triton X100/PBS and multiple exchanges of washing buffer. Samples were then incubated with secondary antibodies (diluted in PB buffer) over-night at RT. Finally, washing steps for 6-8 $\mathrm{h}$ were repeated at RT and samples were mounted in Mowiol with the basal side facing upwards.

\section{Co-Immunostaining of K31 and Trp2 in tail epidermal whole-mounts}

Due to limited choices of primary antibodies suited for tail skin whole-mount preparations, codetection of K31 and Trp2 required sequential immunostaining to avoid cross-reactivity of secondary antibodies (AF647 donkey-anti-goat and AF488 goat-anti-guinea pig, see Table S1). Samples were incubated in secondary antibodies except for AF488 goat anti-guinea pig over-night at RT, followed by $6-8 \mathrm{~h}$ of washing in $0.5 \%$ Triton X-100/PBS and subsequent incubation with AF488 goat anti-guinea pig antibodies over-night. Using this protocol, crossreactivity with Trp2 primary antibodies could be strongly diminished though not completely eliminated. In some samples, dim signals for MCs were noted when acquiring K31 signals. This was attributed to secondary antibodies, as we could validate that the anti-K31 antibody itself does not recognize MC antigens (S.C. Baess, unpublished observation). Importantly, K31 signals solely served to identify scale areas, but not to quantify signal intensities or cell morphologies, hence this weak MC signal was not relevant for the analyses presented in this study.

\section{Immunohistochemistry of ear epidermal whole-mounts}

Preparation and staining of mouse ear epidermis whole-mounts was performed as previously described (Ross et al., 1998). Briefly, mouse ears were depilated with Veet Hair Removal Cream and residual ventral cartilage was removed. Ears were split while floating on PBS, and for further use only the inner ear skin was kept. To separate dermis and epidermis, the skin was placed on $0.5 \mathrm{M}$ ammonium thiocyanate for $25 \mathrm{~min}$ at $37^{\circ} \mathrm{C}$ with the dermal side down. After removing the dermis from the epidermis using forceps, the epidermis was spread on the bottom of a 12-well plate, covered with ice-cold acetone and fixed on ice for $20 \mathrm{~min}$. Next, ear tissues were blocked in $3 \%$ milk/PBS for $2 \mathrm{~h}$ at RT and subsequently incubated with primary antibodies over-night at $4^{\circ} \mathrm{C}$. The next day, ear epidermis was washed three times in PBS for 20 min at RT, followed by incubation with secondary antibodies (diluted in $A B$ buffer, $0.4 \%$ $\mathrm{BSA}, 0.5 \%$ Triton-X100 in PBS) for $2 \mathrm{~h}$ at $37^{\circ} \mathrm{C}$. Finally, the ear tissues were washed three times in PBS at RT and then mounted in Mowiol, with the basal side facing upwards. 


\section{Microscopy}

Confocal images were acquired using a Leica SP8 confocal laser scanning microscope and LASX software using a PL Apo 10x/0.40 CS2 air objective.

\section{Analysis of MC:immunocyte distribution in tail epidermal whole-mounts}

Immunostaining for the scale IFE marker K31 was used to distinguish scale from interscale IFE. Using the free image processing software FIJI, a mask was created based on K31 immunostaining only considering depilated but otherwise intact scale:interscale units for the analysis. Immunostainings for Trp2 (MCs) and Langerin (LCs) were merged with the scale:interscale mask for further compartment-specific quantification of MC or LC numbers. For analysis of MC or LC distribution in Id2Ctrl/KO (Fig. 2B), FVB/N, C57BL/6 WT (Fig. 2D) and partially MC-free tail epidermis of C57BL/6 mice (Fig. 2G), percentages of the total MC or LC population per scale:interscale unit localizing to either scale or interscale IFE were calculated.

For analysis of MC distribution in control and K14ALef1 mice, the mask of the scale IFE (based on K31 immunostaining) was either decreased to $60 \%$ of its size to mark the center region of the scale IFE, or expanded to $110 \%$ to span the scale IFE and its periphery. Remaining K31negative IFE was considered as the third zone (interscale). Percentages of the total MC population per scale:interscale unit localizing to one of the 3 defined regions was calculated per scale:interscale unit. Analysis of MC:LC distribution included at least 6 scale:interscale units per animal.

\section{Analysis of MC:immunocyte density in ear epidermal whole-mounts}

For quantification of MC, LC and DETC density in the ear epidermis, respective cell numbers were counted and density per $\mathrm{mm}^{2}$ was calculated. At least $0.825 \mathrm{~mm}^{2}$ per animal were analysed.

\section{Antibodies}

Details on the antibodies used in this study are listed in Table S1.

\section{Statistical analyses}

Statistical analyses were performed using GraphPad Prism software (GraphPad, version 6.0). Significance was determined as indicated in the figure legends. N-values refer to biological replicates (=mice), are specified in the figure legends and correspond to the sample size used to derive statistics. P-values are ranged as follows: ${ }^{*}, p<0.05 ;{ }^{* *}, p<0.01 ;{ }^{* * *}, p<0.001,{ }^{* * * *}$, $p<0.0001$ as detailed in the figure legends. For all experiments, measurements were taken from minimum three independent biological samples. 


\section{Software}

For data analysis, the following software has been used: GraphPad PRISM VI, Inkscape, ImageJ/Fiji (Rueden et al., 2017; Schindelin et al., 2012), Cell Profiler (Carpenter et al., 2006).

\section{ACKNOWLEDGEMENTS}

We thank the CECAD imaging facility and UoC animal facilities for important services, and Florian Kuester and CMMC Imaging facility for assisting with mouse analysis. We are grateful to Wendy Havran $(\dagger)$ and Deborah Witherden for advice on DETC immunostaining. We acknowledge Kim B. Jensen (University of Copenhagen, Danstem, Denmark) for sharing Lrig1-CreERT2/EGFP mice. We thank all members of the Iden laboratory for stimulating discussions, and Sabrina Cappello and Ann-Kathrin Burkhart for critical feedback on the manuscript.

\section{COMPETING INTERESTS}

The authors declare no competing interests.

\section{FUNDING}

This work was supported by the Deutsche Forschungsgemeinschaft (DFG, German Research Foundation) (grants SPP1782-ID79/2-2 to S.I.; Projektnummer 73111208-SFB 829, A03 to C.N., A10 to S.I.) and the Center for Molecular Medicine Cologne (CMMC; project grants to C.N. and to S.I.). Part of this work was funded by a donation of U. Lehmann to MZ. Work in the Iden laboratory was further supported by the Saarland University, SFB1027, and Excellence Initiative of the German federal and state governments (CECAD Cologne).

DATA AVAILABILITY

Correspondence and requests for materials related to this study should be sent to sandra.iden@uks.eu. All data supporting the findings of this study are available within the paper and its supplemental information, or from the corresponding author on reasonable request.

\section{AUTHOR CONTRIBUTIONS}

Conceptualization, methodology, validation: S.B., A.G., S.I.; investigation: S.B., S.I.; formal analysis: S.B., S.I.; resources: S.I.; visualization: S.B.; mouse models: S.I., C.N., K.S., M.Z.; writing (original draft): S.B., S.I.; writing (review, editing): S.I.; supervision and funding acquisition: S.I.. All authors provided input to the manuscript and data. 


\section{REFERENCES}

Bergstresser, P. R., Toews, G. B. and Streilein, J. W. (1980). Natural and perturbed distributions of Langerhans cells: Responses to ultraviolet light, heterotopic skin grafting, and dinitrofluorobenzene sensitization. J. Invest. Dermatol. 75, 73-77.

Blanpain, C. and Fuchs, E. (2006). Epidermal Stem Cells of the Skin. Annu. Rev. Cell Dev. Biol. 22, 339-373.

Braun, K. M. (2003). Manipulation of stem cell proliferation and lineage commitment: visualisation of labelretaining cells in wholemounts of mouse epidermis. Development 130, 5241-5255.

Breathnach, A. S. and Wyllie, L. M.-A. (1965). Melanin in Langerhans Cells. J. Invest. Dermatol. 45, 401-403.

Burkhart, C. G. and Burkhart, C. N. (2005). The mole theory: Primary function of melanocytes and melanin may be antimicrobial defense and immunomodulation (not solar protection). Int. J. Dermatol. 44, 340-342.

Cao, T., Ueno, H., Glaser, C., Fay, J. W., Palucka, A. K. and Banchereau, J. (2007). Both langerhans cells and intestinal DC cross-present melanoma antigens and efficiently activate antigen-specific CTL. Eur. J. Immunol. 37, 2657-2667.

Carpenter, A. E., Jones, T. R., Lamprecht, M. R., Clarke, C., Kang, I. H., Friman, O., Guertin, D. A., Chang, J. H., Lindquist, R. A., Moffat, J., et al. (2006). CellProfiler: image analysis software for identifying and quantifying cell phenotypes. Genome Biol. 7, R100.

Chang-Rodriguez, S., Hoetzenecker, W., Schwärzler, C., Biedermann, T., Saeland, S. and Elbe-Bürger, A. (2005). Fetal and neonatal murine skin harbors Langerhans cell precursors. J. Leukoc. Biol. 77, 352-360.

Cho, J. S., Modlin, R. L., Miller, L. S., Cho, J. S., Pietras, E. M., Garcia, N. C., Ramos, R. I., Farzam, D. M., Monroe, H. R., Magorien, J. E., et al. (2010). Staphylococcus aureus infection in mice IL-17 is essential for host defense against cutaneous Staphylococcus aureus infection in mice. 120, 1762-1773.

Chuong, C. M. and Noveen, A. (1999). Phenotypic determination of epithelial appendages: Genes, developmental pathways, and evolution. J. Investig. Dermatology Symp. Proc. 4, 307-311.

Cicero, A. Lo, Delevoye, C., Gilles-Marsens, F., Loew, D., Dingli, F., Guéré, C., André, N., Vié, K., Van Niel, G. and Raposo, G. (2015). Exosomes released by keratinocytes modulate melanocyte pigmentation. Nat. Commun. 6, 1-8.

D’Orazio, J., Jarrett, S., Amaro-Ortiz, A. and Scott, T. (2013). UV radiation and the skin. Int. J. Mol. Sci. 14, $12222-12248$.

de Jong, M. A. W. P., de Witte, L., Taylor, M. E. and Geijtenbeek, T. B. H. (2010). Herpes Simplex Virus Type 2 Enhances HIV-1 Susceptibility by Affecting Langerhans Cell Function. J. Immunol. 185, 1633-1641.

Deckers, J., Hammad, H. and Hoste, E. (2018). Langerhans cells: Sensing the environment in health and disease. Front. Immunol. 9, 1-14.

Denat, L., Kadekaro, A. L., Marrot, L., Leachman, S. A. and Abdel-Malek, Z. A. (2014). Melanocytes as instigators and victims of oxidative stress. J. Invest. Dermatol. 134, 1512-1518.

Dias Gomes, M. and Iden, S. (2021). Orchestration of tissue-sclae mechanics and fate decisions by polarity signalling. EMBO J. 40, 1-19.

Didierjean, L., Wrench, R. and Saurat, J. H. (1983). Expression of Cytoplasmic Antigens Linked to Orthokeratosis During the Development of Parakeratosis in Newborn Mouse Tail Epidermis. Differentiation 23, 250-255.

Fitch, K. R., McGowan, K. A., Van Raamsdonk, C. D., Fuchs, H., Lee, D., Puech, A., Hérault, Y., Threadgill, D. W., De Angelis, M. H. and Barsh, G. S. (2003). Genetics of dark skin in mice. Genes Dev. 17, $214-228$.

Gillespie, J. P., Kanost, M. R. and Trneczek, T. (1997). Biological Mediators of Insect Immunity. Annu. Rev. Entomol. 42, 611-643.

Girardi, M., Glusac, E., Filler, R. B., Roberts, S. J., Propperova, I., Lewis, J., Tigelaar, R. E. and Hayday, A. C. (2003). The Distinct Contributions of Murine T Cell Receptor ( TCR ) $\square \square$ २ and TCR ๑૫२ T Cells to Different Stages of Chemically Induced Skin Cancer The Journal of Experimental Medicine. 198, 747-755.

Glover, J. D., Knolle, S., Wells, K. L., Liu, D., Jackson, I. J., Mort, R. L. and Headon, D. J. (2015). Maintenance of distinct melanocyte populations in the interfollicular epidermis. Pigment Cell Melanoma Res. 28, 476-480.

Gomez, C., Chua, W., Miremadi, A., Quist, S., Headon, D. J. and Watt, F. M. (2013). The interfollicular epidermis of adult mouse tail comprises two distinct cell lineages that are differentially regulated by Wnt, Edaradd, and Lrig1. Stem Cell Reports 1, 19-27.

Gonzales, K. A. U. and Fuchs, E. (2017). Skin and Its Regenerative Powers: An Alliance between Stem Cells 
and Their Niche. Dev. Cell 43, 387-401.

Graham, D. J. G. J., Tiffany, S. M. and Vogel, F. S. (1978). The Toxicity of Melanin Precursors. J. Invest. Dermatol. 70, 113-116.

Hacker, C., Kirsch, R. D., Ju, X. S., Hieronymus, T., Gust, T. C., Kuhl, C., Jorgas, T., Kurz, S. M., RoseJohn, S., Yokota, Y., et al. (2003). Transcriptional profiling identifies Id2 function in dendritic cell development. Nat. Immunol. 4, 380-386.

Havran, W. L. and Jameson, J. M. (2010). Epidermal T Cells and Wound Healing. Bone 184, 5423-5428.

Hemmi, H., Yoshino, M., Yamazaki, H., Naito, M., Iyoda, T., Omatsu, Y., Shimoyama, S., Letterio, J. J., Nakabayashi, T., Tagaya, H., et al. (2001). Skin antigens in the steady state are trafficked to regional

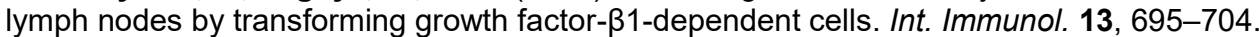

Hirobe, T. (1984). Histochemical survey of the distribution of the epidermal melanoblasts and melanocytes in the mouse during fetal and postnatal periods. Anat. Rec. 208, 589-594.

Hong, Y., Song, B., Chen, H.-D. and Gao, X.-H. (2015). Melanocytes and Skin Immunity. J. Investig. Dermatology Symp. Proc. 17, 37-39.

Jameson, J., Ugarte, K., Chen, N., Yachi, P., Fuchs, E., Boismenu, R. and Havran, W. L. (2002). A role for skin $ү \delta$ T cells in wound repair. Science (80-. ). 296, 747-749.

Kaminski, M. J., Cruz, P. D., Bergstresser, P. R. and Takashima, A. (1993). Killing of Skin-derived Tumor Cells by Mouse Dendritic Epidermal T-Cells. Cancer Res. 53, 4014-4019.

Kobayashi, M., Asano, H., Fujita, Y. and Hoshino, T. (1987). Development of ATPase-positive, immature Langerhans cells in the fetal mouse epidermis and their maturation during the early postnatal period. Cell Tissue Res. 248, 315-22

Köhler, C., Nittner, D., Rambow, F., Radaelli, E., Stanchi, F., Vandamme, N., Baggiolini, A., Sommer, L., Berx, G., van den Oord, J. J., et al. (2017). Mouse Cutaneous Melanoma Induced by Mutant BRaf Arises from Expansion and Dedifferentiation of Mature Pigmented Melanocytes. Cell Stem Cell 21, 679-693.

Kunisada, T., Yamazaki, H., Hirobe, T., Kamei, S., Omoteno, M., Tagaya, H., Hemmi, H., Koshimizu, U., Nakamura, T. and Hayashi, S. I. (2000). Keratinocyte expression of transgenic hepatocyte growth factor affects melanocyte development, leading to dermal melanocytosis. Mech. Dev. 94, 67-78.

Le Poole, I. C., Mutis, T., Van den Wijngaard, R. M. J. G. J., Westerhof, W., Ottenhoff, T., De Vries, R. R. P. and Das, P. K. (1993a). A novel, antigen-presenting function of melanocytes and its possible relationship to hypopigmentary disorders. J. Immunol. 151, 7284-7292.

Le Poole, I. C., Van Den Wijngaard, R. M. J. G. J., Westerhof, W., Verkruisen, R. P., Dutrieux, R. P., Dingemans, K. P. and Das, P. K. (1993b). Phagocytosis by normal human melanocytes in vitro. Exp. Cell Res. 205, 388-395.

Leclercq, G. and Plum, J. (1995). Stimulation of TCR V gamma 3 cells by gram-negative bacteria. J. Immunol 154, 5313-5319.

Li, M., Knapp, S. K. and Iden, S. (2020). Mechanisms of melanocyte polarity and differentiation: What can we learn from other neuroectoderm-derived lineages? Curr. Opin. Cell Biol. 67, 99-108.

Li, Z., Lamb, R., Coles, M.C., Bennett, C.L., and Ambler, C.A. (2021) Inducible ablation of CD11C+ cells to determine their role in skin wound repair. Immunology. 163, 105-111.

Liu, Y., Xue, L., Gao, H., Chang, L., Yu, X., Zhu, Z., He, X., Geng, J., Dong, Y., Li, H., et al. (2019). Exosomal miRNA derived from keratinocytes regulates pigmentation in melanocytes. J. Dermatol. Sci. 93, 159-167.

MacLeod, A. S., Hemmers, S., Garijo, O., Chabod, M., Mowen, K., Witherden, D. A. and Havran, W. L. (2013). Dendritic epidermal T cells regulate skin antimicrobial barrier function. J. Clin. Invest. 123, 43644374.

Mishima, Y. (1966). Melanosomes in phagocytotic vacuoles in Langerhans cells. J. Cell Biol. 30, 417-423.

Niemann, C., Owens, D. M., Hülsken, J., Birchmeier, W. and Watt, F. M. (2002). Expression of $\Delta$ NLef1 in mouse epidermis results in differentiation of hair follicles into squamous epidermal cysts and formation of skin tumours. Development 129, 95-109.

Nishimura, E. K. (2011). Melanocyte stem cells: A melanocyte reservoir in hair follicles for hair and skin pigmentation. Pigment Cell Melanoma Res. 24, 401-410.

Page, M. E., Lombard, P., Ng, F., Göttgens, B. and Jensen, K. B. (2013). The epidermis comprises autonomous compartments maintained by distinct stem cell populations. Cell Stem Cell 13, 471-482.

Park, S., Matte-Martone, C., Gonzalez, D. G., Lathrop, E. A., May, D. P., Pineda, C. M., Moore, J. L., Boucher, J. D., Marsh, E., Schmitter-Sánchez, A., et al. (2021). Skin-resident immune cells actively coordinate their distribution with epidermal cells during homeostasis. Nat. Cell Biol. 23, 476-484. 
Ray, S. and Lechler, T. (2011). Regulation of asymmetric cell division in the epidermis. Cell Div. 6, 1-6.

Ross, R., Gillitzer, C., Kleinz, R., Schwing, J., Kleinert, H., Förstermann, U. and Reske-Kunz, A. B. (1998). Involvement of NO in contact hypersensitivity. Int. Immunol. 10, 61-69.

Rueden, C. T., Schindelin, J., Hiner, M. C., DeZonia, B. E., Walter, A. E., Arena, E. T. and Eliceiri, K. W. (2017). ImageJ2: ImageJ for the next generation of scientific image data. BMC Bioinformatics 18, 529.

Sada, A., Jacob, F., Leung, E., Wang, S., White, B. S., Shalloway, D. and Tumbar, T. (2016). Defining the cellular lineage hierarchy in the interfollicular epidermis of adult skin. Nat. Cell Biol. 18, 619-631.

Sánchez-Danés, A., Hannezo, E., Larsimont, J.-C. C., Liagre, M., Youssef, K. K., Simons, B. D. and Blanpain, C. (2016). Defining the clonal dynamics leading to mouse skin tumour initiation. Nature 536, 298-303.

Schindelin, J., Arganda-Carreras, I., Frise, E., Kaynig, V., Longair, M., Pietzsch, T., Preibisch, S., Rueden, C., Saalfeld, S., Schmid, B., et al. (2012). Fiji: An open-source platform for biological-image analysis. Nat. Methods 9, 676-682.

Schreurs, M. W. J., Eggert, A. A. O., De Boer, A. J., Vissers, J. L. M., Van Hall, T., Offringa, R., Figdor, C. G. and Adema, G. J. (2000). Dendritic cells break tolerance and induce protective immunity against a melanocyte differentiation antigen in an autologous melanoma model. Cancer Res. 60, 6995-7001.

Schuhmachers, G., Ariizumi, K., Mathew, P. A., Bennett, M., Kumar, V. and Takashima, A. (1995). 2B4, a new member of the immunoglobulin gene superfamily, is expressed on murine dendritic epidermal $T$ cells and plays a functional role in their killing of skin tumors. J. Invest. Dermatol. 105, 592-596.

Schweizer, J. and Marks, F. (1977). Developmental study of the distribution and frequency of Langerhans cells in relation to the formation of patterning in mouse tail epidermis. J. Invest. Dermatol. 69,198-204

Seré, K., Baek, J. H., Ober-Blöbaum, J., Müller-Newen, G., Tacke, F., Yokota, Y., Zenke, M. and Hieronymus, T. (2012). Two Distinct Types of Langerhans Cells Populate the Skin during Steady State and Inflammation. Immunity 37, 905-916.

Sheng, J., Chen, Q., Wu, X., Dong, Y. W., Mayer, J., Zhang, J., Wang, L., Bai, X., Liang, T., Sung, Y. H., et al. (2021). Fate mapping analysis reveals a novel murine dermal migratory langerhans-like cell population. Elife 10, 1-27.

Stoitzner, P., Tripp, C. H., Eberhart, A., Price, K. M., Jung, J. Y., Bursch, L., Ronchese, F. and Romani, N. (2006). Langerhans cells cross-present antigen derived from skin. Proc. Natl. Acad. Sci. 103, 7783-7788.

Su, Q. and Igyártó, B. Z. (2019). Keratinocytes Share Gene Expression Fingerprint with Epidermal Langerhans Cells via mRNA Transfer. J. Invest. Dermatol. 139, 2313-2323.e8.

Tobin, D. J. (1998). A possible role for Langerhans cells in the removal of melanin from early catagen hair follicles. Br. J. Dermatol. 138, 795-798.

Tripp, C. H., Chang-rodriguez, S., Stoitzner, P., Holzmann, S., Stössel, H., Douillard, P., Saeland, S., Koch, F. and Elbe-bu, A. (2004). Ontogeny of Langerin / CD207 Expression in the Epidermis of Mice. J. Inve Dermatology Dermatology 122, 670-672.

Tsuruta, D., Kaneda, K., Teramae, H. and Ishii, M. (1999). In vivo activation of Langerhans cells and dendritic epidermal T cells in the elicitation phase of murine contact hypersensitivity. Br. J. Dermatol. 140, 392-399.

Upadhyay, P. R., Ho, T. and Abdel-Malek, Z. A. (2021). Participation of keratinocyte- and fibroblast-derived factors in melanocyte homeostasis, the response to UV, and pigmentary disorders. Pigment Cell Melanoma Res. 34, 762-776.

Urabe, K., Aroca, P., Tsukamoto, K., Mascagna, D., Palumbo, A., Prota, G. and Hearing, V. J. (1994). The inherent cytotoxicity of melanin precursors: A revision. BBA - Mol. Cell Res. 1221, 272-278.

Van Raamsdonk, C. D., Fitch, K. R., Fuchs, H., De Angelis, M. H. and Barsh, G. S. (2004). Effects of Gprotein mutations on skin color. Nat. Genet. 36, 961-968.

Watt, F. M. and Collins, C. A. (2008). Role of $\beta$-catenin in epidermal stem cell expansion, lineage selection, and cancer. Cold Spring Harb. Symp. Quant. Biol. 73, 503-512.

Xiang, J., Qiu, M. and Zhang, H. (2020). Role of Dendritic Epidermal T Cells in Cutaneous Carcinoma. Front. Immunol. 11, 1-6.

Yokota, Y., Mansouri, A. and Mori, S. (1999). Developmentof peripheral lymphoid organsand natural killercells dependsonthe helix-loop-helix inhibitor Id2. Nature 397, 702-706. 


\section{FIGURE LEGENDS}

Figure 1: Mutually exclusive localization of MCs and epidermis-resident immunocytes to scale and interscale IFE compartments of murine tail epidermis. (A) Micrographs of tail epidermis whole-mounts from 3-months old wild-type C57BL/6 mice, immunostained for markers of LCs (Langerin), MCs (Trp2/Dct), and scale IFE (K31). Representative for $n=4$. Scale bar: $75 \mu \mathrm{m}$. (B) Immunostaining of tail epidermis whole-mounts from 3-months old wildtype C57BL/6 mice for Langerin and DETC marker $\gamma \delta T C R$. Representative for $n=3$. Scale bar: $300 \mu \mathrm{m}$. (C) Immunostaining of tail epidermis whole-mounts from wild-type C57BL/6 mice for Trp2, K31, and LC markers MHCII (P5, P10) or Langerin (P21). Representative for $n=3$. Scale bars: $75 \mu \mathrm{m}$. (D) Schematic illustration of scale:interscale IFE patterns and associated structures and cell types in murine tail epidermis; a: anterior, $\mathrm{p}$ : posterior, $\mathrm{s}$ : scale, is: interscale.

Figure 2: LCs and MCs localize independently of each other to distinct intraepidermal niches. (A) Langerin, Trp2 and K31 immunostaining of tail epidermis whole-mounts from 3months old control and Id2KO mice. Scale bar: $100 \mu \mathrm{m}$. (B) Quantification of A; MC distribution per scale:interscale unit (\% of MC). MC numbers in each compartment (scale, interscale) were normalized to the total $M C$ number per scale:interscale unit. $n=4$; ns: $p=0.8370$; mean \pm s.d.; one-way ANOVA/Tukey's multiple comparisons test. (C) Langerin, Trp2 and K31 immunostaining of tail epidermis whole-mounts of 3-months old C57BL/6 and FVB/N mice. Scale bar: $250 \mu \mathrm{m}$. (D) Quantification of C; MC distribution to scale and interscale IFE (\% of $\mathrm{MC}$ ). MC numbers in each compartment (scale, interscale) were normalized to the total MC number per scale:interscale unit. $n=3$; $n s: p=0.8755$; mean \pm s.d.; one-way ANOVA/Tukey's multiple comparisons test. (E) Quantification of C; LC distribution per scale:interscale unit (\% of LC). LC numbers in each compartment (scale, interscale) were normalized to the total LC number per scale:interscale unit. $n=3$; $n s: p=0.8186$; mean \pm s.d.; one-way ANOVA/Tukey's multiple comparisons test. (F) Representative images of tail skin from 3-months old wild-type C57BL/6 with partial spontaneous loss of MCs. Left: intact tail skin. Right: epidermis wholemount. Scale bar: $3000 \mu \mathrm{m}$ (top), $1500 \mu \mathrm{m}$ (bottom). (G) Immunostaining for Langerin, Trp2 and K31. Tail epidermis whole-mounts of 3-months old wild-type C57BL/6 mice with spontaneous loss of MCs (center) in posterior tail region. Scale bar: $250 \mu \mathrm{m}$. (H) Quantification of G; LC distribution per scale:interscale unit (\% of LC) in MC-free and MC-containing tail epidermis of C57BL/6 mice. LC numbers in each compartment (scale or interscale) were normalized to the total LC number per scale:interscale unit. $n=4$; ns: $p=0.3429$; mean \pm s.d.; one-way ANOVA/Tukey's multiple comparisons test. s: scale, is: interscale. 
Figure 3: The MC:immunocyte distribution dynamically adapts to changes of epidermal scale:interscale patterning in Lrig1KO mice. (A) Langerin, Trp2 and K31 immunostaining of tail epidermis whole-mounts from 3-months old control and Lrig1KO mice. Representative for $n=3$. Scale bars: $500 \mu m$. (B) MHCII, Trp2 and K31 immunostaining of tail epidermis wholemounts from control and Lrig1KO mice at P10. Representative for $n=4$. Scale bar: $100 \mu \mathrm{m}$. (C) $\mathrm{MHCII}$ and $\gamma \delta \mathrm{TCR}$ immunostaining of tail epidermis whole-mounts from control and Lrig1KO mice at $P 10$. Representative for $n=4$. Scale bar: $100 \mu$ m. s: scale, is: interscale

Figure 4: Repression of epidermal Wnt signaling causes ectopic localization of MCs to interscale IFE. (A) Immunostaining of tail epidermis whole-mounts of 3-months old control and K14 $\triangle$ NLef1 mice for Langerin, Trp2 and K31. Representative for $n=6$. Scale bar: $100 \mu \mathrm{m}$. (B) Illustration of one scale:interscale unit with corresponding categorization into scale center, scale periphery and interscale IFE for analysis of MC distribution in K14 $\Delta$ NLef1 mice as done in C. (C) Quantification of MC distribution per scale:interscale unit (\% of LCs) in control and $\mathrm{K} 14 \Delta$ NLef1 mice. MC numbers per compartment were normalized to the total number of the

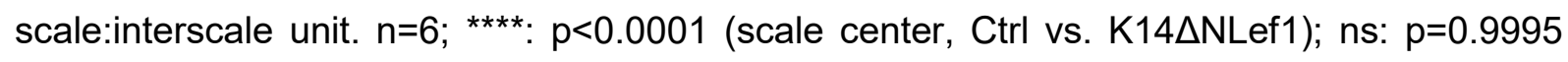
(scale periphery, Ctrl vs. K14 $\Delta$ NLef1); ${ }^{* * * *}: \mathrm{p}<0.0001$ (interscale IFE, Ctrl vs. K14 $\Delta$ NLef1); mean \pm s.d.; one-way ANOVA/Tukey's multiple comparisons test. (D) Trp2-immunostaining of ear epidermis whole-mounts of 3-months old control and K14 $\Delta$ NLef1 mice. Nuclei were counterstained using DAPI. Scale bar: $250 \mu \mathrm{m}$. (E) Quantification of D; MC density (MC number $/ \mathrm{mm}^{2}$ ) in ear epidermis. $n=5 ;{ }^{* *}: p=0.0079$; mean \pm s.d.; Mann-Whitney test. (F) Trp2, $\mathrm{K} 31$ and $\mathrm{MHCll}$ immunostaining of tail epidermis whole-mounts of control and K14 $\triangle$ NLef1 mice at $P 5$ and P10. Representative for $n=3$. Scale bar: $100 \mu m$. (G) Trp2, K31, and Langerin immunostaining of tail epidermis whole-mounts from P21 control and K14ANLef1 mice. Representative for $n=3$. Scale bar: $100 \mu \mathrm{m}$. $(\mathrm{H}) \gamma \delta \mathrm{TCR}$ and Langerin immunostaining of tail epidermis whole-mounts from 3-months old control and K14 $\Delta$ NLef1 mice. Representative for $\mathrm{n}=4$. Scale bar: $250 \mu \mathrm{m}$. s: scale, is: interscale. 
Baess et al. Figure 2

\section{A Trp2 Langerin}
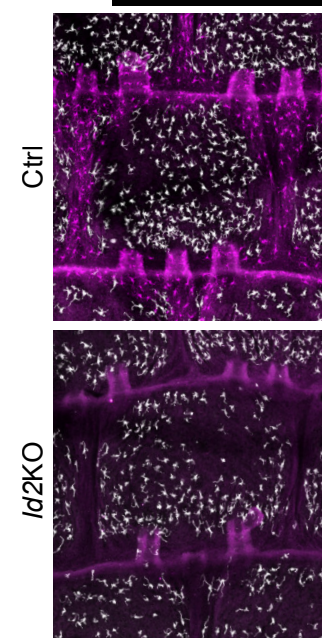

C

Trp2 Langerin
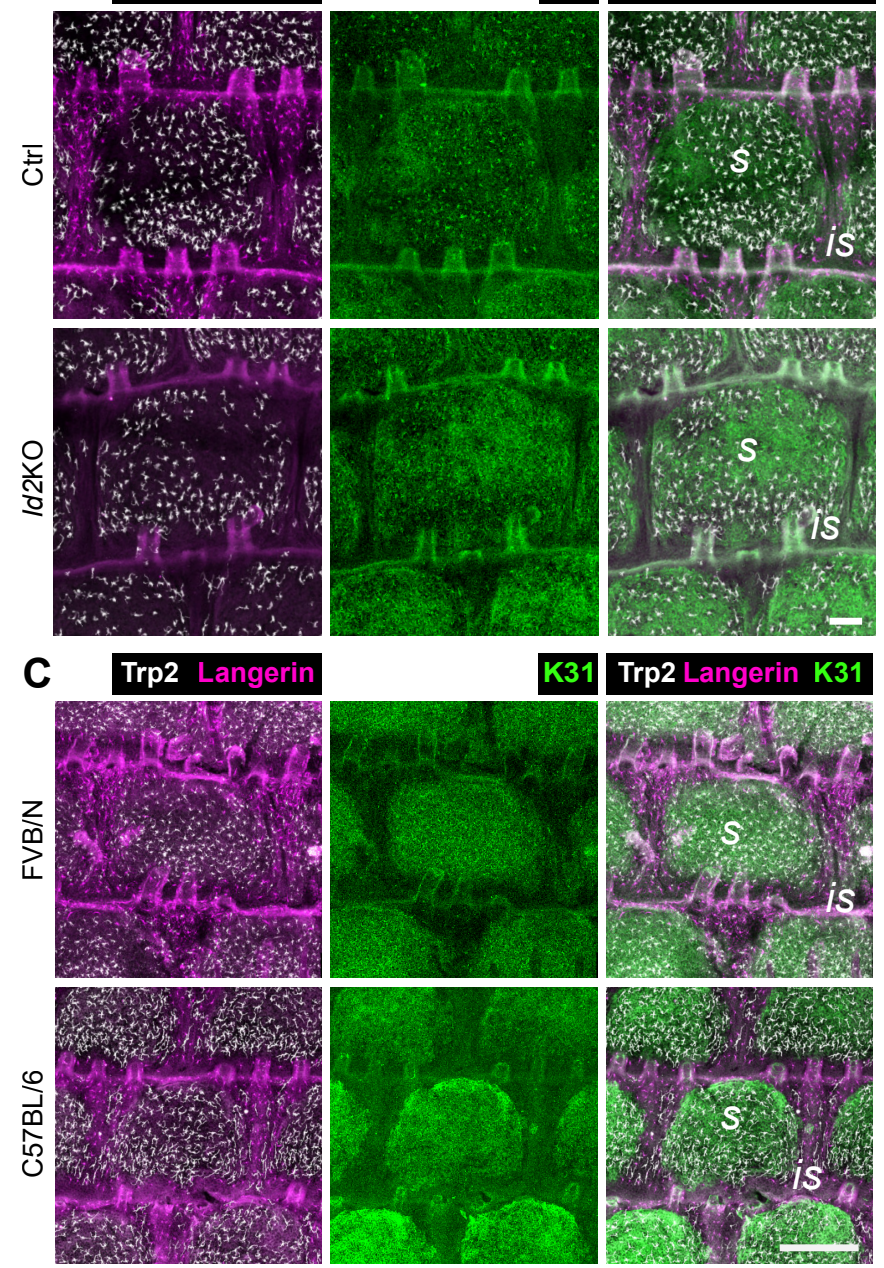

F

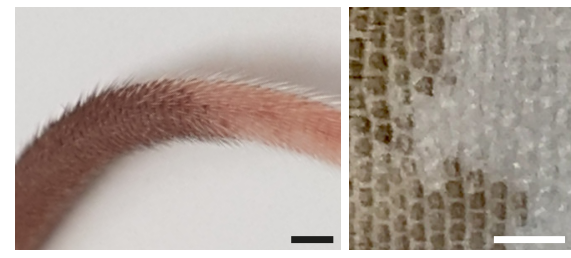

31 Trp2 Langerin K31

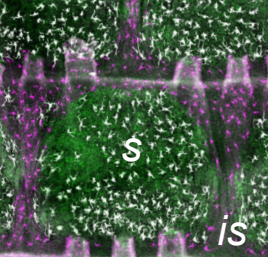

B MC distribution in Control and Id2KO mice

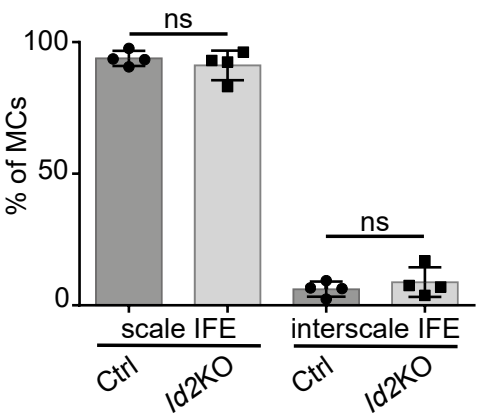

D $\quad$ MC distribution in differentially pigmented mice

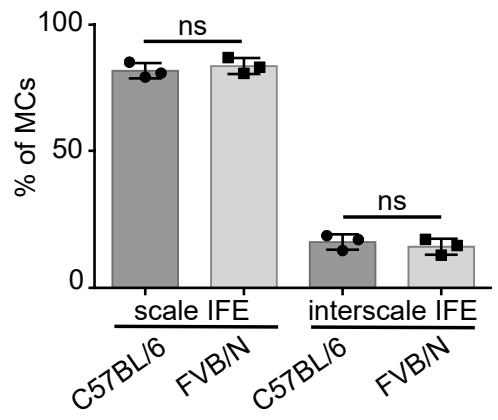

E

LC distribution in differentially pigmented mice

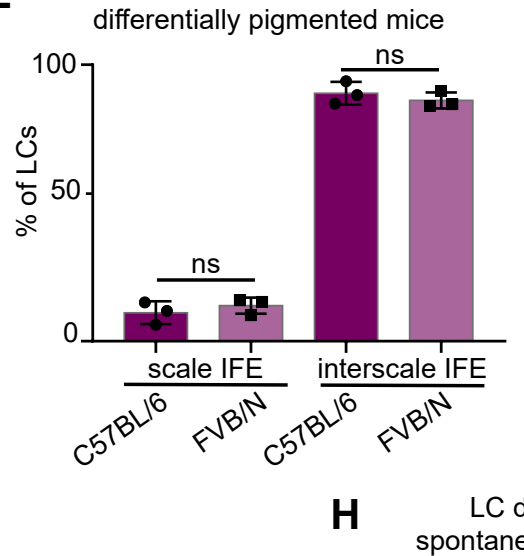

H LC distribution upon spontaneous loss of IFE MCs
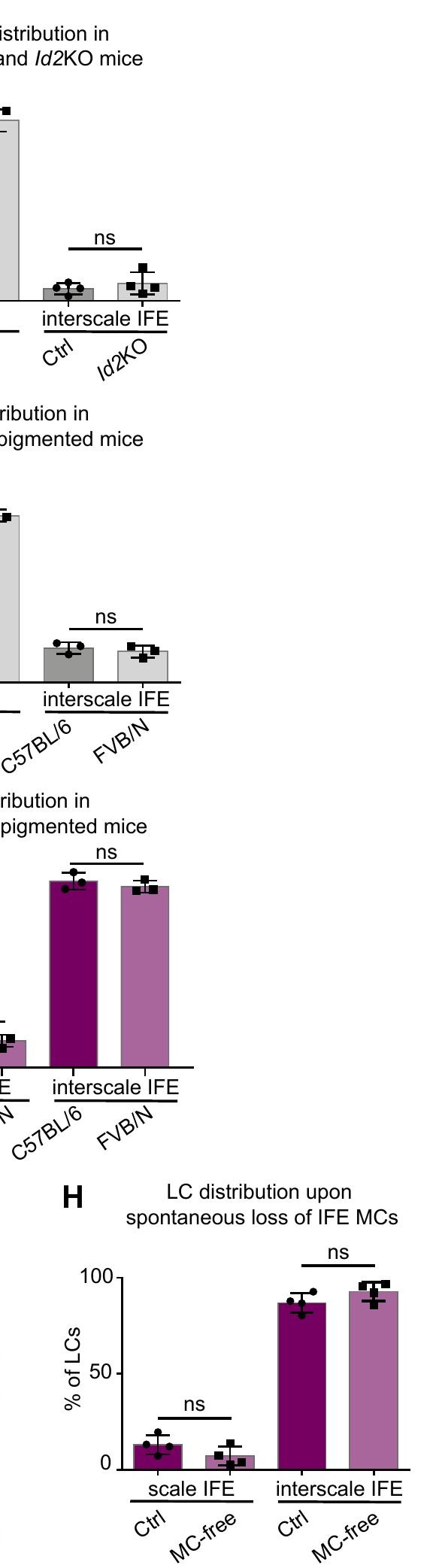

E

$$
\text { distribution upon }
$$

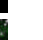

\section{G}

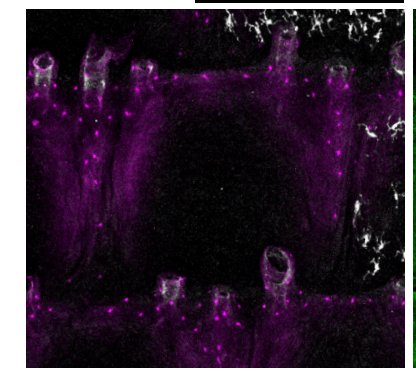

K31

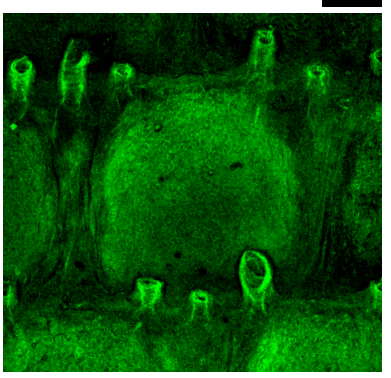

Trp2 Langerin K31

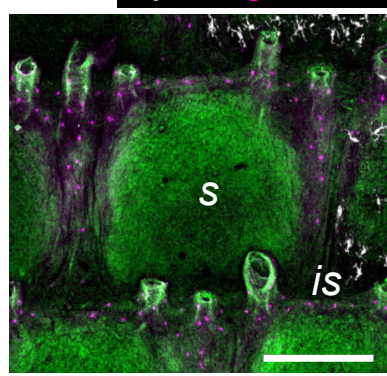


Baess et al. Figure S3
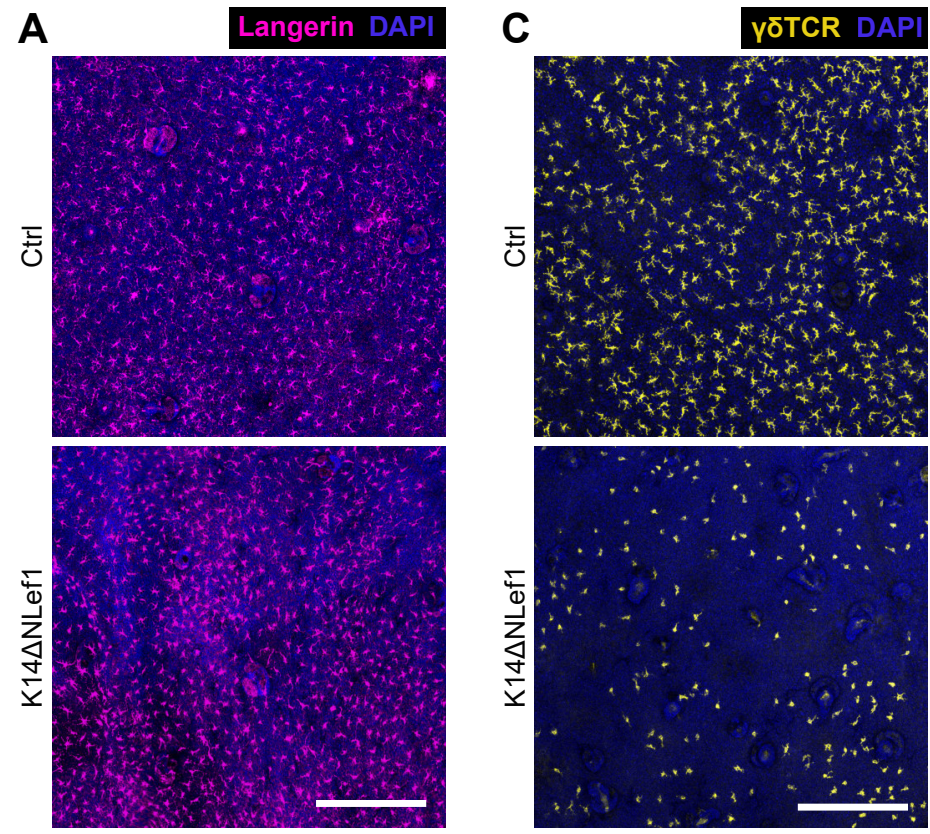

B LC density
in ear epidermis

D

DETC density in ear epidermis
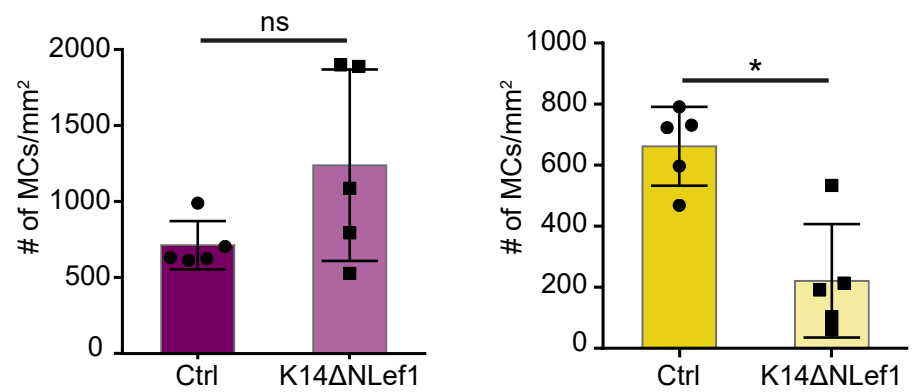

Figure S3: Reduced DETC numbers in ear epidermis of K14DNLef1 mice. (A) Langerin immunostaining of ear epidermis whole-mounts of 3-months old control and $\mathrm{K} 14 \Delta$ NLef1 mice. Nuclei were counterstained using DAPI. Scale bar: $250 \mu \mathrm{m}$. (B) Quantification of A; LC density (LC number $/ \mathrm{mm}^{2}$ ) in ear epidermis. $n=5$; ns: $p=0,2222$; mean \pm s.d.; Mann-Whitney test. (C) y $\delta T C R$ immunostaining of ear epidermis whole-mounts of 3-months old control and K14 $\mathrm{NL}$ ef1 mice. Nuclei were counterstained using DAPI. Scale bar: $250 \mu \mathrm{m}$. (D) Quantification of C; DETC density (DETC number/mm²) in ear epidermis. $n=5 ;{ }^{*}: p=0,0159 ;$ mean \pm s.d.; Mann-Whitney test. 
Baess et al. Figure S4

A

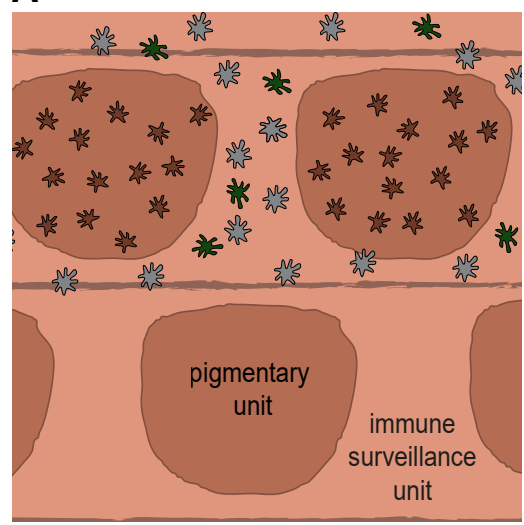

interscale IFE

Ctrl

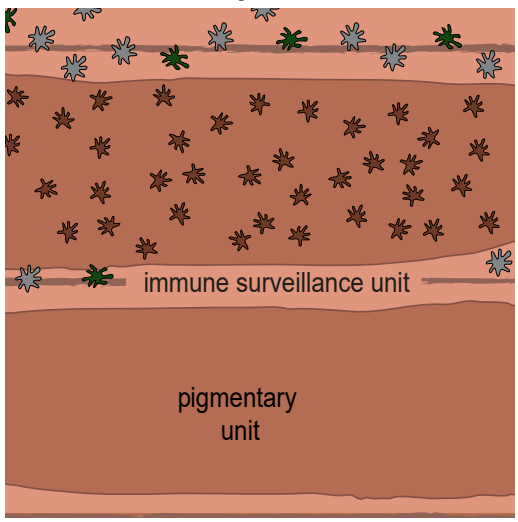

净 melanocyte 资 Langerhans cell
K14 N NLef1

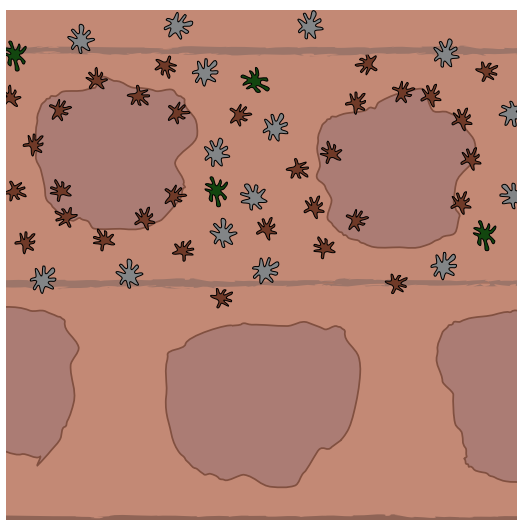

औरs dendritic epidermal T cell

Figure S4: Schematic summary of Lrig1- and Wnt-dependent partitioning of MCs and resident immunocytes into distinct epidermal niches. (A) Epidermal niches and their resident cell types in control mice (left), Lrig1KO mice exhibiting scale IFE fusion (middle), and $\mathrm{K} 14 \Delta \mathrm{NLef1}$ mice with MC localization inverted to interscale IFE, resulting in loss of functional segregation of pigmentary units and immune surveillance in mouse tail skin (right panel). 


\section{Baess et al.}

Supplementary Table 1: Antibodies and imaging reagents used in this study

\begin{tabular}{|c|c|c|c|c|}
\hline \multicolumn{5}{|l|}{ Primary antibodies } \\
\hline antibody (supplier) & catalog nr. & clone/Ref. & species & dilution \\
\hline K31 (Progen) & GP-HHA1 & polyclonal & guinea pig & IF 1:100 \\
\hline $\begin{array}{l}\text { Trp2 (Santa Cruz } \\
\text { Biotechnology) }\end{array}$ & sc-10451 & polyclonal & goat & IF 1:100 \\
\hline Langerin (Invitrogen) & $14-2073-80$ & eBioRMUL.2 & rat & IF 1:100 \\
\hline $\begin{array}{l}\text { MHC II (M. Pasparakis lab, } \\
\text { CECAD, Cologne) }\end{array}$ & - & - & rat & IF 1:100 \\
\hline \multicolumn{5}{|l|}{ Secondary antibodies } \\
\hline $\begin{array}{l}\text { goat anti-guinea pig AF488 } \\
\text { (Invitrogen) }\end{array}$ & A11073 & polyclonal & goat & IF 1:500 \\
\hline $\begin{array}{l}\text { Donkey anti-rat AF594 } \\
\text { (Dianova) }\end{array}$ & $712-585-153$ & polyclonal & donkey & IF 1:500 \\
\hline $\begin{array}{l}\text { Donkey anti-goat AF647 } \\
\text { (Invitrogen) }\end{array}$ & A21447 & polyclonal & donkey & IF 1:500 \\
\hline \multicolumn{5}{|l|}{ Other reagents } \\
\hline DAPI (Carl Roth) & - & - & - & IF 1:500 \\
\hline \multicolumn{5}{|c|}{ Directly-conjugated antibodies } \\
\hline $\begin{array}{l}\text { FITC anti-mouse TCR } \gamma / \delta \\
\text { (BioLegend) }\end{array}$ & 118105 & GL3 & $\begin{array}{l}\text { armenian } \\
\text { hamster }\end{array}$ & IF 1:300 \\
\hline
\end{tabular}

